\title{
Detener el cambio climático. Una labor de los profesores de ciencias
}

To stop the climatic change. A work of the professors of sciences

\author{
Rómulo Gallego Badillo1, Royman Pérez Miranda', Adriana Patricia Gallego Torres ${ }^{2}$ y Roberto \\ Enrique Figueroa Molina ${ }^{3}$
}

${ }^{1}$ Profesores de la Universidad Pedagógica Nacional. Bogotá, D. C. Colombia. ${ }^{2}$ Profesora de la Universidad Distrital Francisco José de Caldas. Bogotá, D. C. Colombia. ${ }^{3}$ Profesor de La Universidad del Atántico. Barranquilla, Colombia. Grupo de Investigación Representaciones y Conceptos Cient́ficos - Grupo IREC. rgallego@pedagogica.edu.co rovman@pedagogica.edu.co adpgallegot@udistrital.edu.co roberfigue@hotmail.co

\section{Resumen}

La civilización actual se halla amenazada por el problema del cambio dimático. Este problema es consecuencia del incremento incontrolado de la concentración de los gases de invernadero en la atmósfera, principalmente del $\mathrm{CO}_{2}$. Hay que detener el incremento de esa concentración. En esta "cruzada" han de tomar partido todos los profesores en general, y los profesores de ciencias en particular, lo que implica convertir el modelo científico que explica las causas y consecuencias de ese cambio, en objeto de enseñanza en los programas académicos de formación inicial y continua de profesores.

\section{Palabras clave}

Cambio dimático, efecto invernadero, historia, epistemología, didáctica de las ciencias y formación de profesores.

\section{Abstract}

Today's civilization is threatened by dimate change. This problem is a consequence of the uncontrolled increase in the concentration of greenhouse gases in the atmosphere, mainly $\mathrm{CO2}$. We must stop the increase of that concentration. In this campaign all are expected to take part, especially science teachers. This implies converting scientific model that explains the causes and consequences of that change, in the main subject of education in the academic training of preservice and in-service teachers.

\section{Key Words}

Climate change, greenhouse gases, history, science philosophy, science education and teachers' education.

\section{Introducción}

El modelo científico para la comprensión de las causas y consecuencias del cambio dimático, debido al aumento creciente e incontrolado de los gases de efecto invernadero que si bien tiene una larga historia de construcción, tan solo a partir de 2004 ha sido admitido por la comunidad cientúfica intemacional, y paulatinamente por cada uno de los gobiemos actuales del planeta. El proceso de admisión del modelo, que es un ejemplo reciente para explicar la historia intema y externa de las ciencias (Laudan, 2005), se enfrentó tanto a las concepciones epistemológicas dominantes, como a los intereses de los magnates de las industrias petrolíferas y carboníferas. Con 
respecto a estas últimas, crearon la "Coalición Global del Clima', un grupo patrocinado, entre otros, por Chevron, Chrysler, Exxon, Ford, General Motors, Mobil, Shell y Texaco; grupo que invirtió alrededor de 13 millones de dólares en su campaña publicitaria contra el Protocolo de Kyoto. Patrocinó, además, los denominados escépticos del calentamiento global, quienes se dedicaron a la publicación de libros en los que desvirtuaban los resultados obtenidos sobre la realidad de dicho cambio dimático y sus consecuencias (Kolbert, 2008).

En lo tocante a las concepciones epistemológicas dominantes originadas en el tradicional dominio de las aproximaciones epistemológicas positivista de corte experimentalista, en razón de que quienes se ocuparon del problema del cambio dimático y en razón de que no eran factibles experimentos en tiempo real (Humphreys, 2007), decidieron diseñar modelos computacionales para contrastar sus hipótesis sobre los efectos del aumento de la temperatura global terrestre (Flannery, 2008). Fue con base en esta tradición y en una versión no crítica del experimentalismo, las que aprovecharon los creadores de la "Coalición Global del Clima" (Kolbert, 2008), para producir la convicción de que esos resultados eran en sí no admisibles, no confiables.

Otras razones que se adujeron en contra partieron de los fundamentos de la dinámica de los sistema no lineales. Un acudir que no puso de presente las consecuencias epistemológicas introducidas en la concepción de naturaleza por esta dinámica. En este sentido, se dejó de lado el análisis del problema del no cumplimiento de las relaciones directas de causalidad; del hecho de que estos sistema no siguen esquemas repetitivos ni pautas discemibles (Hayles, 1993); de que rompen la simetría temporal que las relaciones entre causa y efecto, que se apartan de la causalidad derivada de la dinámica newtoniana (Prigogine y Stenger, 1990a: 1990b; Briggs y Peat 1992; Cruchfield, 1987; Hayles, 1993). Se aprovecharon del hecho de que esa dinámica no lineal aun no ha sido objeto de enseñanza.

\section{Las consecuencias del cambio climático}

Son variadas las publicaciones que dan cuenta de este problema y advierten sobre lo que sucederá si no se toman medidas urgentes para detener el creciente aumento de la concentración de los gases de efecto invernadero en la atmósfera del planeta (Kolvert, 2008; Lovelock, 2007; Weart, 2006; Flannery, 2008; Gore, 2007). Sin embargo, los resultados de las investigaciones y predicciones contrastadas a través de las simulaciones virtuales, no se han convertido aún en objeto de enseñanza, a pesar de las consecuencias que ya se están sufiiendo, como son en el caso de los países que no se hallan en los hemisferios en los que se presentan las auatro estaciones, por ejemplo, Colombia. La tradicional sucesión de épocas de lluvias y de sequías se ha alterado considerablemente, sin que las explicaciones que se dan hayan acudido al problema del cambio dimático. Parecería ser que se entienden desde la perspectiva habitual de un mandato divino y no de las consecuencias del actuar de los seres humanos sobre la naturaleza.

El problema de hacer del cambio dimático objeto dem trabajo en el aula, implica un cambio en las concepciones histónicas, epistemológicas y didácticas acerca de las relaciones entre las ciencias y las tecnologías que se desprendieron de la de la denominada revolución científica del siglo XVII (Vessuri, 1992; Restivo, 1992). Se inició la ampliación de las ciudades y complejización de la vida urbana; situación esta en la que históricamente se creo una negativa valoración de la naturaleza (Carvalho, 2000). Los desarrollos de la relación entre ciencia y tecnología, ligada a la lógica del capitalismo emergente y los resultados obtenidos, explicativamente contribuyeron al dominio de la versión de conocimiento cientúfico-tecnológico que aún sigue siendo el modelo que, de manera acrítica, domina y es el que se hace objeto de enseñanza.

Para comprender lo postulado, hay que recurrir a la historia interna y externa para sostener que el gran éxito de la ciencia moderna fue la simplificación de la complejidad; éxito este por el que se 
está pagando el precio de la crisis ambiental. El método que se inventó, dentro de la aproximación epistemológica positivista, parceló la naturaleza y en su concepción de objetividad postuló la necesidad de dejar a un lado las valoraciones propias de los seres humanos. La ciencia se dividió en disciplinas y se creó el convencimiento de una ciencia neutral y libre de valores. La racionalidad se hizo equivalente a "racionalidad científica" y el conocimiento, "conocimiento científico" el único conocimiento reconocible; una convicción epistemológica con la que se desvaloró otras formas de conocer y de saber del ser humano en el mundo (Funtowics y De March, 2000), entre ellas la milenaria de los indígenas americanos.

Es necesario, para comprender el problema del cambio dimático y su conversión en ciencia escolar, de una mirada tanto intemalista como extemalista del mismo, y de un cambio en la versión epistemológica desde la cual se han valorado como dudosos los resultados de las predicciones obtenidas con los modelos computacionales. La predicción dave es la de que cuando la concentración de $\mathrm{CO}_{2}$ en la atmósfera planetaria sea de 500 partes por millón (ppm), se alcanzará el punto de no retomo y sobrevendrá la catástrofe.

\section{Algunas notas sobre el uso de los combustibles}

El uso de los derivados del petróleo se hizo posible a partir del momento en que en Italia, los físicos Barsante y Mateucai, en 1854, diseñaran el primer motor de explosión interna. Agréguese a lo anterior que, 1886, K. Benz patenta su automóvil de tres ruedas. Retornando a los comienzos del siglo XX, H. Ford, con su famoso modelo "T"' y desde la "producción en cadena" que en términos de la "administración cientúfica" tayloriana y siguiendo la lógica de producción capitalista, masificó paulatinamente el uso del automóvil, y con ello el consumo de los derivados del petróleo. La emisión cada vez creciente de los combustibles fósiles, tanto por los automóviles como por la industria basada en la combustión de estos combustibles fósiles, es, según la versión de los partidarios del problema del calentamiento global, un problema que afecta el mercado de sus inversiones en investigaciones sobre el desarrollo de la química como una de las ciencias de la naturaleza.

No es posible dejar por fuera el incremento en la producción y uso de los llamados biocombustibles, término este que puede llamar a equívocos, sobre todos entre las personas que no han tenido una alfabetización científica y tecnológica básica. En efecto, por ejemplo, el etanol obtenido es un compuesto biodegradable. El problema es cuando se mezda con gasolina, para su combustión en los motores de vehículos u otras máquinas, puesto que es convertido en $\mathrm{CO}_{2}$ que va a parar a la atmósfera, contribuyendo a la concentración de ese gas. Además, la producción de biocombustibles tiene otras consecuencias nefastas para la población de menores recursos, ya que está encareciendo en el mercado los precios de los cereales. El aumento de la hambruna es indiscutible.

Los líderes mundiales en producción de etanol son Brasil, Estados Unidos, Francia, Gran Bretaña y España. Estados Unidos emplea mayoritariamente maíz, Brasil caña de azúcar y las destileńas europeas trigo, cebada y azúcar de remolacha. Colombia ha entrado en esta dinámica con la conversión del azúcar de caña, desde la misma racionalidad financiera de la lógica del mismo capitalismo que ha producido la posible emergencia planetaria a la que se enfrenta el mundo actual, y en la que los países del denominado Tercer Mundo o países en desarrollo, su contribución al cambio dimático es relativa (Brown, 2008). 


\section{Biodegradación y residuos biodegradables}

En el movimiento y la conciencia internacional sobre la conservación del medio ambiente, hay una creciente preocupación por el uso, en este caso, de bolsas y botellas que llevan el rótulo de "Biodegradables". Es algo que es indispensable celebrar, aplaudir y estimular, aun cuando todavía no sea un comportamiento generalizado. Pero čnasta dónde la mayoría de las personas ha construido un concepto sobre la biodegradación o de aquello que se quiere significar con residuos biodegradables?

Biodegradación quiere decir que todos los componentes de la naturaleza en sus procesos de descomposición entran a la gran cadena alimenticia, siendo los microorganismos quizás los últimos en intervenir en el proceso que restaura cada uno de los sistemas dinámicos de no equilibrio que relacionados unos con otros conforman el medio ambiente planetario. En este sentido, todo lo que la biosfera produce es biodegradable. Se trata de un mecanismo de disipación de las perturbaciones creados para restaurar cada uno de esos sistemas estacionarios de no equilibrio; mecanismos este que, además fue y ha sido producto de millones de años de evolución de la vida sobre el planeta.

Tanto desde el punto de vista químico como bioquímico, los procesos de biodegradación requieren, fuera de los microorganismos implicados con sus enzimas y demás, de unas condiciones de, por lo menos temperatura y humedad, sin los cuales la biodegradación como proceso carece de las correspondientes condiciones iniciales. La mirada desde la química y la bioquímica puntualiza que como proceso, la biodegradación transcurre en el tiempo; un postulado básico para comprender la cinética de los procesos enzimáticos 0 , si se quiere, la velocidad a la que transcurren dichas reacciones. En consecuencia, cada material o residuo natural es biodegradado en un tiempo determinado por la correspondiente cinética de las reacciones enzimáticas. Por supuesto que en la naturaleza ese tiempo se inscribe en el promedio de vida de los microorganismos que llevan a cabo la biodegradación final.

Esto mecanismos biológicos de disipación se vieron trastornados auando los químicos al servicio de la gran industria de los derivados del petróleo y desde el triunfo de la química orgánica desarrollada en Alemania, crearon los procesos de síntesis de los plásticos o, mejor de polímeros conformados por cadenas de carbono e hidrógeno. La producción de bolsas, botellas y toda clase de embalajes elaborados a partir de estos polímeros se perfeccionó industrialmente, lo que tuvo como consecuencia una disminución en los costos de producción. Se celebró la ventaja que conllevaba su uso y este se generalizó, desplazando las tradicionales prácticas del uso del papel y demás formas de embalaje.

Las consecuencias para el medio ambiente han sido desastrosas, por cuanto los microorganismos que evolutivamente habían desarrollado los procesos bioquímicos para la degradación de los residuos naturales, no contaban con los mecanismos enzimáticos para convertir estos polímeros en sustento alimenticio. Desde cuando en tiendas y supermercados se instauró el empleo de estas bolsas y las botellas mediante las cuales se expenden las bebidas carbonatadas, producida por polimerización de los derivados del petróleo, el medio ambiente se fue polucionando con el creciente aumento de tales residuos arrojados indiscriminadamente al ambiente.

Se reitera aquí que esas bolsas y botellas fabricadas con polímeros derivados del petróleo, según estudios realizados, las primeras tardan 400 años y las segundas 500 años en ser biodegradadas, aun cuándo la pregunta que se pone en el tapete de la discusión es ¿Qué sistema de disipación de perturbaciones del planeta tienra se encarga de restaurar la perturbación del sistema estacionario de no equilibrio que constituye el ambiente terrestre; ayya restitución tiene que ver con la supervivencia de la especie humana? En la actualidad se están elaborando estos 
envases a partir del polímero obtenido del ácido láctico (PLA, por su sigla en inglés) y la materia prima es el maíz común. La fórmula del ácido láctico es $\mathrm{CH}_{3} \mathrm{CHOHCOOH}$, por lo que el polímero no son solo enlaces carbono hidrógeno, lo que lo hace biodegradable. Para que estos residuos sean biodegradado en el lapso de entre cuatro y seis semanas hay que "comportarlos" con el fin de que al alcanzar esta mezda los 65 grados Celsius las bacterias actúen. De otra manera el tiempo de biodegradación es más largo.

Para una mayor comprensión es necesario hacer aquí una breve lección de química. Supóngase que en una solución relativamente diluida de doruro de hidrógeno en agua (Ácido dorhídrico) se introduce un trozo entero de hierro, un davo o puntilla por ejemplo, la reacción de formación del doruro de hierro comienza, pero esta procede con una velocidad bastante lenta, hasta el punto que pueden transaurrir muchas horas para que todo el hierro sea consumido. Otra hecho se presenta arando en vez del davo se agregan limaduras de hierro, la reacción transcurre rápidamente.

Hágase ahora una analogía con las bolsas y las botellas plásticas, lo mismo con las bolsas de PLA. Sí todos estos residuos no son cortados, triturados y reducidos a pedazos suficientemente pequeños, entonces cuando son indiscriminadamente arrojados al ambiente, para su biodegradación se requerirá posiblemente mucho más tiempo que el señalado. Cuando las empresas que se ocupan del recidaje de las bolsas y botellas plásticas, es este el procedimiento que usualmente emplean para volver a reutilizar el polímero del con el que se han elaborado. Para la "compostación" de las bolsas fabricadas con PLA hay que proceder de manera análoga. En consecuencia, cuando se habla de biodegradación es indispensable precisar las condiciones en las arales este proceso se hace posible, incluso y más allá, identificar biológicamente cada una de las especies de micro organismos que se encargan de dicha biodegradación.

Dado el también interés educativo, la pregunta recurrente es ¿Qué se enseña al respecto, en los sistemas educativos, sobre la biodegradación?, ces esta una palabra que desde la perspectiva bachelardiana no es más qué un término de uso social?, chasta qué punto los didactas de las ciencias y los que se han encargado de la asignatura de Educación Ambiental han hecho objeto de enseñanza las implicaciones conceptuales y metodológicas que conlleva la biodegradación y la admisión de bolsas y botellas fabricadas con polímeros biodegradables? Se reitera, por tanto lo de una alfabetización cientíica y tecnológica para todos, que ha de ser esencial en los programas curriculares de educación básica y media, por encima de la resolución de pruebas estandarizadas, ayyas críticas se han puntualizado en este texto.

\section{Encuentros, cumbres y reuniones internacionales}

En junio de 1992, las Naciones Unidas celebraron en Río de Janeiro la llamada Cumbre de la Tierra. Acudieron algo más de veinte mil personas y representantes de la mayoría de los países. Las discusiones condujeron a aprobar el Convenio Marco de las Naciones Unidas sobre el Cambio Climático que comprometía a los signatarios a reducir sus emisiones de $\mathrm{CO}_{2}$ a los niveles de concentración determinados en 1990 . Se sucedieron posteriormente varias rondas, como la de Berlín en 1995, la de Ginebra en 1996 y, finalmente, la de Kyoto en diciembre de 1997. El acuerdo surgido en la última sesión en esta ciudad japonesa, no pasó de ser solo un agregado al Convenio Marco de Río de Janeiro. Recibió el título de Protocolo de Kioto para el convenio Marco de Naciones Unidas sobre el Cambio Climático (Kolbert, 2008).

El Protocolo de Kyoto no fue firmado por Australia, Estados Unidos, Liechtenstein y Mónaco. Este Protocolo ha generado una división entre quienes basados en las predicciones de los modelos computacionales se hallan convencidos de que el cumplimiento de sus recomendaciones es esencial para la supervivencia de la actual civilización, y quienes se oponen radicalmente allegando razones estrictamente económicas y políticas. Los cambios urgentes que se requieren arestan mucho 
menos que las inversiones hechas para reparar los daños causados por los efectos del calentamiento global. Los acuerdos de este Protocolo generaron las reacciones en contra del mismo, por parte de los industriales de los combustibles, quienes presionaron para que el Senado de los Estados Unidos votara en contra cualquier política conducente a la reducción de las emisiones de $\mathrm{CO}_{2}$.

En octubre de 2002, frente al problema del derretimiento de los casquetes polares, en Alaska se reunieron funcionarios de las ocho naciones con territorios en el Ártico, Estados Unidos, Rusia, Canadá, Dinamarca, Noruega, Suecia, Finlandia e Islandia. Discutieron sobre los efectos del cambio dimático y del calentamiento global en esta región del planeta. Se acordó un plan de estudio con una asignación de dos millones de dólares. En noviembre de 2004, en Reikiavik, se presento un documento mamotrético y burocrático que no pasó de ser solo esto. Sobre lo acontecido en la reunión de Poznam, Polonia, en el que se busca un nuevo pacto para frenar el cambio climático; reunión esta que es preparativa para la próxima conferencia de Copenhague en el 2009. Se discutieron las distintas soluciones cient́fico-tecnológicas necesarias para no alcanzar ese hipotético punto de no retorno. Terminó sin mayores acuerdos decisivos.

\section{La formación de profesores de ciencias}

T. S. Kuhn (1972), desde 1962 puso de presente que los profesores de ciencia enseñan una ciencia que ya pasó y que hace parte de la historia, sin acudir a las metodologías propias de los historiadores. A lo anterior hay que agregar que llevan al aula una "ciencia producto", descontextualizada, por lo que las actuales problemáticas sociales, culturales y medioambientales causadas por los productos de las investigaciones cient́fico-tecnológicas no se analizan durante esa enseñanza. Por tanto, se transmite una concepción de ciencia que es independiente de la cultura y que hace que ella no sea en sí "cultura", aras de una objetividad y universalidad mal planteada.

En el caso del modelo cientúfico para el problema del cambio dimático, si bien ha sido aceptado, aún esta en un proceso de desarrollo conceptual y metodológico, por lo que su didactización ha de partir de este convencimiento, como también del de que su transposición ha de acudir a la historia tanto intema como externa de su formulación. Como se ha discurrido en la presente ponencia, requiere igualmente de una comprensión de la dinámica de los sistemas no lineales e, induso, en la de que el ambiente ha de ser trabajado desde la misma perspectiva, por lo que hay que hablar de complejidad ambiental (Leff, 2000; Lewin, 1995).

Dado que son variadas las disciplinas científicas desde las auales se ha formulado el modelo de la referencia, no se propone que se introduzca en los currículos y planes de estudio asignaturas como "Cambio Climático I, II.., ya que este problema ha de atravesar toda la propuesta. Este problema como objeto de enseñanza ha de ser inscrito en las relaciones ciencia, tecnología, sociedad, ambiente y cultura (CTSAC). Se persigue, en consecuencia una formación del profesorado de ciencias en la actualidad del desarrollo de un hecho científico, con las consecuencias catastróficas que se avecinan (Kolbert, 2008), que posibilite una enseñanza de las ciencias comprometida con la supervivencia de la civilización actual y de la especie humana.

\section{Conclusiones}

Lo que se ha dicho hasta el presente, se encuentra en los contenidos de algunos de los libros de divulgación. Los autores de esta ponencia son del parecer de que la comunidad de didactas de las ciencias, como los de las otras ciencias, tienen necesariamente que ocuparse de introducir en los programas académicos de formación inicial y continua de profesores del problema del cambio dimático y del aumento de la temperatura global del planeta tierra. Se trata de un cambio cultural 
que retome críticamente esa cultura ancestral nuestra, para la cual la tiena que pertenecemos indisolublemente. Nuestros abuelos americanos la denominaron "Pacha Mama", la madre tierra (Gómez, 2000; Mejía, 2008) o "Haba", o "Ati" o "Puinawai" (Garzón y Román, 2008).

\section{Bibliografía}

Humphreys, P. (2007). Epistemología del siglo XXI. Revista Antropos. Huellas del conocimiento, No. 214, 65-70.

Kolbert, E. (2008). La catástrofe que viene. Barcelona: Planeta.

Laudan, R. (2005). La "nueva" historia de la ciencia: implicaciones para la filosofía de la ciencia. En: S. F. Martínez y G. Guillaumin (Ed.), Historia, filosofía y enseñanza de la ciencia pp. 121 - 130. México: UNAM. Alianza.

Prigogine, I y Stenger, I (1990a). La nueva aliarza. Metamorfosis de la ciencia. Madrid:

Prigogine, I. y Stenger, I (1990b). Entre el tiempo y la eternidad. Madrid: Teanos. 